\title{
Drought resistance in some Prunus persica (L.) Batsch cultivars damaged with Plum Pox Virus
}

\author{
Brailko V.A.*, Mitrofanova I.V., Mitrofanova O.V., Chirkov S.V., Mesyats N.V. \\ Order of the Red Banner of Labour Nikita Botanical Gardens - National Scientific Center of the RAS, \\ Yalta, Russia \\ *e-mail:valentina.brailko@yandex.ru
}

Prunus persica (L.) Batsch is one of the most valuable and widely cultured fruit crops, which is characterized by a rather high yield and resistance to abiotic stress factors. Nowadays, the great problem of this culture is its high degree of disease and pest damages. In this regard, a comparative analysis of peach cultivars, infected with Plum pox virus (PPV), resistance to air and soil drought on the Southern coast of the Crimea has been made. On the leaf blades of the damaged plants, narrow, indistinct stripes were observed in the form of a pattern, rarely-rings of light yellow or yellow color and leaf wrinkling. The degree of the tree crown damages was different: from $10 \%$ ('Podarok Neveste') to $80 \%$ ('Lakomyiy', 'Pushistyiy Ranniy', 'Mechta'). The leaf blades in the damaged plants had a number of structural differences compared with asymptomatic ones: leaf thickness reduction, palisade index decrease (by 8-12\%, noted in 'Lakomyiy' and 'Krymskiy Shedevr'), formation of large intercellular spaces in spongy tissue, necrotic processes in palisade chlorenchyma, a greater amount of crystal inclusions in cell cytoplasm. Due to the cultivar differences, in optimal conditions of the growing season beginning, cultivars demonstrated the water regime changes because of the infection were noted: 'Mechta', 'Pushistyiy Ranniy', 'Cardinal'. Their water retention capacity significantly reduced. The summer drought led to a decrease of the total water content in the leaf tissues. In asymptomatic plants water content was 59-70 \%; in damaged plants it reduced to $55 \%$ ('Karnavalnyiy', 'Pushistyiy Ranniy', 'Ambergold'), water deficit was $12-26 \%$ in healthy plants and $20-31 \%$ - in damaged plants. High drought tolerance is generally characteristic of the cultivars: 'Krymskiy Shedevr', 'Dixired', 'Tulpan', 'Podarok Neveste'. PPV damages reduces the resistance to abiotic stress in the summer period in 'Karnavalnyiy', 'Pushistyiy Ranniy', 'Demerdzhinskiy'.

Acknowledgements: This work was supported by the Russian Science Foundation, project No. 19-16-00091. 